Cad.Est.Ling., Campinas, (40):77-92, Jan./Jun. 2001

\title{
CONCORDÂNCIA DE GÊNERO NO PROCESSAMENTO DE FORMAS PRONOMINAIS
}

\author{
LETÍCIA MARIA SICURO CORRÊA \\ (PUC/RJ)
}

\begin{abstract}
Pronominal agreement and grammatical agreement are distinguished. In particular, it is argued that pronominal agreement may involve the semantic interpretation of the formal gender feature of the pronoun independently from the interpretation of the gender feature of the antecedent. The vulnerability of lexicalsyntactic information concerning the gender of the antecedent is also considered. Two experiments are reported which explore the possibility of [+animate] nouns being gender invariable in Portuguese and information is provided regarding the natural gender (sex) of the gender invariable antecedent of the pronoun. The effect of grammatical gender/natural gender congruence upon the processing of pronominal forms is investigated. In the fist experiment, the sentences of the pair are contiguous and in the second one, the distance between antecedent and pronoun is enlarged. The results of Exp. 1 suggest that lexicalsyntactic information prevails though the processing of pronominal reference is facilitated when there is semantic information compatible with the semantic interpretation of the gender of the pronoun. The results of Exp 2 suggest that pronominal reference can be solved on a semantic basis when information concerning the grammatical gender of the linguistic antecedent is not available. The results are compatible with a model of sentence processing which predicts integrative processes at the end of the sentence. They also contribute to the creation of a discourse-processing model that expresses how cohesive relationships involving pronominal forms can be established.
\end{abstract}

\section{INTRODUÇÃO}

Este estudo focaliza a concordância de gênero na compreensão de formas pronominais em contexto discursivo. Concordância pode ser entendida como uma relação de dependência sintática e/ou semântica que se expressa morfologicamente de modo a refletir a compatibilidade de propriedades lexicais relativas a gênero, número e pessoa, entre os termos da relação. Essa caracterização se aplica tanto à concordância gramatical (entre determinante/nome; nome/adjetivo; sujeito/verbo) quanto à concordância pronominal. No entanto, esses processos são eminentemente distintos. Este estudo começa por caracterizar a diferença entre esses dois tipos de relação e por apresentar as implicações dessa diferença para o processamento de formas pronominais.

$\mathrm{O}$ processamento de formas pronominais tem gerado um grande número de estudos na literatura psicolingüística nas últimas décadas (Crawley, Stevenson \& Kleiman 1990; Gordon \& Chan 1995; Grosz \& Sidner 1986; McKoon, Greene \& Ratcliff 1993; Arnold, Eisenband, Brown-Schmidt \& Trueswell 2000). Contudo, pouco se sabe sobre o modo como sentenças são processadas no discurso de modo que 
relações coesivas, tais como as que se mantém por meio de formas pronominais, se estabeleçam. O presente estudo explora as bases lingüísticas e as condições de processamento em que a referência pronominal pode se estabelecer. Consideram-se, em particular, o tipo de informação necessária para que a concordância pronominal de gênero se estabeleça e a possibilidade de o gênero do pronome ser semanticamente interpretado independentemente do gênero do antecedente. Considera-se, ainda, a possibilidade de a referência pronominal se resolver em bases estritamente semânticas quando da perda de informação léxico-sintática relativa ao gênero do antecedente lingüístico.

\section{CONCORDÂNCIA GRAMATICAL E PRONOMINAL}

Em linhas gerais, pode-se entender concordância gramatical como a expressão morfológica de uma relação de dependência ou vinculação sintática entre elementos de um sintagma ou oração. Do ponto de vista da teoria lingüística (Chomsky 1995), essa expressão seria subproduto de uma operação geral de checagem de traços formais de elementos do léxico, no curso de uma derivação lingüística. A checagem de traços estaria relacionada com o conceito de interpretabilidade, entendido, a princípio, como uma propriedade de traços formais relativa à possibilidade de estes serem semanticamente interpretados na interface da língua com sistemas de desempenho. Nomes teriam traços formais, intrínsecos ou opcionais, de pessoa, número e gênero, semanticamente interpretáveis na interface, enquanto que determinantes, adjetivos e verbos teriam traços não interpretáveis a serem checados com aqueles no curso da derivação lingüística. A presença/ausência de morfemas flexionais nesses últimos, expressando a relação de concordância, seria resultado de variação paramétrica relativa à força dos traços formais. Os traços ditos fortes promoveriam movimento sintático na chamada sintaxe aberta, acarretando a presença de morfemas flexionais que expressam o valor assumido por um traço formal não interpretável, no nível de interface identificado como Forma Fonética.

Ainda que a noção de interpretabilidade não esteja suficientemente clara no âmbito da teoria lingüística ${ }^{1}$ e apresente problemas para o tratamento do traço intrínseco de gênero de nomes em línguas românicas — o qual não necessariamente encontra correlato semântico (Comrie 1999; Corbett 1991), a interpretabilidade de traços formais do léxico no nível de interface que resulta de uma derivação linguiística apresenta-se como um conceito promissor, a ser explorado na integração de modelos de língua com modelos de processamento lingüístico.

A Psicolingüística busca dar conta do processamento de relações de concordância, em sentido amplo, considerando tanto a concordância gramatical (Friederici \& Jacobsen 1999; Liu, Chiarello \& Quan 1999; Pearlmutter, Garnsey \& Bock 1999; Deutsch \& Bentin 2001; Schriefers \& Jescheniak 1999), quanto relações de

${ }^{1}$ A possibilidade de interpretação semântica não necessariamente implica que um traço formal deva ser caracterizado como interpretável. 
compatibilidade de gênero, número e pessoa, que podem se estabelecer fora dos limites da sentença, como no caso da concordância pronominal (Arnold et al. 2000; Carreiras et al. 1993; Ganham et al. 1995). Contudo, poucos são os estudos que levam em conta as propriedades do material lingüístico sobre o qual um aparato processador de linguagem atuaria, à luz de um modelo de língua.

No caso do processamento em nível intra-sentencial, percebe-se a incorporação de um modelo de língua na distinção que é estabelecida entre informação de natureza léxico-sintática, léxico-semântica e semântica, em sentido mais amplo, no desenvolvimento de modelos de processamento que incorporam componentes modulares (Friederici 1995; Hahne \& Friederici 2000; Friederici et al. 2001; Gunter et al. 2000). O estudo empírico do processamento da concordância vem, assim, facilitar o contraste de previsões oriundas de modelos dessa natureza com as previsões de modelos incrementais (ou integrativos) (Friederici \& Jacobsen 1999; van Berkun et al. 1999).

No que diz respeito ao processamento de formas pronominais no discurso, por outro lado, uma concepção teórica de língua tende a ser ignorada. Na literatura psicolingüística, assume-se que a interpretação de formas pronominais é dependente de procedimentos estratégicos que levam em conta todo o tipo de informação disponível. Informação relativa a gênero é, assim, tomada como uma pista para a identificação do antecedente do pronome (Garnham et al. 1995).

Neste estudo, considera-se que representações de natureza lexico-sintática relativas a gênero, número e pessoa, que se mantém acessíveis na memória de trabalho quando da identificação de uma forma pronominal, podem garantir certo grau de automatismo no processamento da mesma. Contudo, a vulnerabilidade desse tipo de representação pode dificultar o processamento dele dependente. Assim sendo, torna-se crucial considerar o que se apresenta na interface da língua com sistemas de desempenho, no que concerne à visibilidade de informação de natureza léxico-sintática — interpretabilidade semântica e expressão morfofonológica, de modo a caracterizar em que bases lingüísticas o processamento de formas pronominais pode transcorrer. Além disso, é necessário que se considerem as condições de processamento em que esse tipo de informação pode se manter.

No que diz respeito ao processamento da concordância gramatical, resultados experimentais sugerem que este processo envolve o pareamento pós-lexical (ou seja, pós reconhecimento e acesso lexical) dos traços léxico-sintáticos dos termos da relação, cuja representação temporária ficaria mantida na memória de trabalho (Friederici \& Jacobsen 1999). Nesse caso, o pareamento seria função da posição de cada termo numa configuração decorrente do parsing em andamento. Assim, no processamento da concordância no DP (sintagma determinante), o pareamento de traços, entre determinante e nome e entre nome e adjetivo, seria determinado pelo modo como o sistema computacional da língua permitiria ao parser atuar sobre o material lingüístico em processamento. No que concerne à interpretabilidade na interface, apenas os traços do nome com que o determinante e o adjetivo concordam seriam relevantes. Os morfemas flexionais nesses últimos seriam mera expressão da relação sintática que se mantém entre estes e o nome. 
No caso do processamento da concordância pronominal, pronome e antecedente não mantém uma relação sintática entre si e sim uma relação semântica, de coreferência. Logo, a flexão dos pronomes não pode ser expressão morfofonológica de uma relação de checagem de traços, uma vez que esta não se estabelece. Como considerar, então, os traços formais dos pronomes? Como expressão de traços formais com valores opcionais, tal como o traço de gênero da maior parte dos nomes com traço semântico [+ animado] em Português (masculino ou feminino), e como o traço de número de nomes com traço semântico [+ contável] (singular ou plural)? Essas questões remetem a um problema maior relativo ao status gramatical de formas pronominais plenas, para o qual o estudo do processamento lingüístico pode contribuir.

No caso do gênero, assumir que os traços formais do pronome são interpretáveis na interface em função da presença de um determinado morfema significa assumir que o gênero gramatical do pronome irá remeter a uma categoria semântica específica (no caso, sexo feminino e masculino). Restrições à animacidade do antecedente de formas pronominais nominativas seriam compatíveis com essa possibilidade, como nas formas pronominais nominativas no Português Europeu, as quais requerem antecedente [+ animado] ${ }^{2}$. Entretanto, esse tipo de restrição não é geral. Formas pronominais, mesmo as nominativas, podem admitir antecedente [- animado], como (o livro; a estátua, etc.) no Português Brasileiro. Verifica-se também que, nas línguas românicas em geral, formas pronominais podem, em princípio, ter como antecedente um termo [+ animado] ou [+ humano], com traço intrínseco de gênero semanticamente indeterminado, como em $a$ vítima. Nesse caso, o fato de a compatibilidade imediata entre a interpretação semântica do gênero do pronome com o do antecedente linguístico poderia dificultar o processamento da relação de co-referencialidade.

Essas observações sugerem que o processamento da concordância pronominal envolve pareamento de traços léxico-sintáticos, tal como se observa no processamento da concordância gramatical. Contudo, diferentemente desse último, o processamento da concordância pronominal não envolveria qualquer tipo de determinismo sintático entre os termos da relação que garantisse o pareamento de traços formais de representações lexicais. O pareamento de traços formais seria, no caso da concordância pronominal, exclusivamente dependente da disponibilidade de informação léxico-sintática relativa a possíveis antecedentes do pronome na memória de trabalho. Essa dependência pode, não obstante, tornar o processamento de formas pronominais particularmente vulnerável no discurso. Diferentemente da concordância gramatical que se estabelece em domínios sintaticamente definidos, a concordância pronominal pode se estabelecer entre elementos de diferentes sentenças. Não há, a princípio, restrições à distância entre antecedente e pronome. Contudo, o fechamento de uma sentença como unidade de processamento sintático e semântico possibilita o esvaecimento da representação temporária de suas propriedades lexicais e sintáticas da memória de trabalho (Jarvella \& Herman 1972; Townsend \& Bever 1978). Assim sendo, o acesso à informação léxico-sintática relativa aos traços formais do antecedente de uma forma pronominal no

${ }^{2}$ O PE parece não admitir antecedente [- animado] para formas pronominais nominativas. Agradeço a Jairo Nunes, por me ter chamado atenção para esse ponto, e a Maria João Freitas, por ter usado sua intuição de falante de Português Europeu em julgamentos de aceitabilidade da referência pronominal. 
discurso pode ser prejudicado quando antecedente e pronome encontram-se em ambientes sintáticos distintos.

A representação conceptual do referente do antecedente de uma forma pronominal tenderia, por outro lado, a permanecer acessível para além dos limites da sentença em que o antecedente se encontra. No caso de a vítima de terno escuro, por exemplo, o referente dessa expressão referencial pode ser tomado (por inferência) como sendo um homem. Uma vez que a representação literal da expressão referencial a vítima de terno escuro se esvaísse da memória de trabalho, a representação conceptual de um homem de terno escuro que foi vítima de algo permaneceria disponível. Seria possível ao pronome remeter diretamente a esse tipo de representação, sem a intermediação de um antecedente lingüístico? Para isso, os traços formais do pronome teriam de ser, tais como os do nome, interpretados semanticamente na interface. Com base nessa interpretação, uma representação conceptual compatível seria buscada. O uso da referência pronominal na chamada anáfora discursiva ou profunda, em que o pronome faz referência a um elemento atualizado a partir de uma estrutura cognitiva complexa, tal como um esquema, sugere que este pode ser o caso (Gernsbacher 1991).

O processamento da concordância pronominal apresenta, portanto, um quadro complexo. Um pareamento de traços léxico-sintáticos seria necessário, ainda que contingente à disponibilidade da representação léxico-sintática do possível antecedente na memória de trabalho. Em princípio, uma vez estabelecido esse pareamento, o gênero do pronome não seria interpretado. Tem-se, assim, A criança ... Ela, independentemente do sexo da mesma. Contudo, a possibilidade de o traço de gênero do pronome ser semanticamente interpretado viabilizaria o processamento da referência pronominal para a maioria dos nomes com traço semântico [+ animado], quando da perda de informação léxico-sintática relativa ao gênero do antecedente lingüístico. Assim sendo, não é claro até que ponto essa interpretação viria interferir no processamento da referência pronominal em quaisquer condições.

Em estudo do processamento da concordância de gênero realizado com falantes de Italiano, formas pronominais tiveram antecedentes com traço semântico [+ animado]/ [+ humano] e invariante quanto ao gênero, como vítima (Cacciari, Carreiras e Cionini 1997). Contudo, os resultados não são conclusivos quanto à interferência de uma possível interpretação semântica do gênero do pronome no processamento da concordância pronominal. Apresenta-se, abaixo, uma breve revisão dos estudos com Italiano e, em seguida, dois experimentos são relatados em que, diferentemente dos experimentos em Italiano, a congruência entre o gênero gramatical do pronome e o gênero natural do referente do antecedente é manipulada.

\section{GÊNERO GRAMATICAL E CONCORDÂNCIA PRONOMINAL}

Em uma série de experimentos de leitura automonitorada em Italiano, Cacciari, Carreiras e Cionini (1997) utilizaram-se de nomes com traços [+animado], [+humano], invariantes em gênero como o antecedente mais acessível de (i) pronomes nominativos em sentenças independentes, (ii) clíticos acusativos em orações completivas, estando o 
antecedente na oração principal; (iii) nomes próprios em sentenças independentes. As formas tomadas como anafóricas - pronomes plenos, clíticos e nomes próprios; foram variadas em gênero, de modo que só uma delas seria compatível com o gênero gramatical do antecedente. Os resultados apresentaram menor tempo de leitura, ao fim da sentença, para pronomes, clíticos e nomes próprios que possuem o mesmo gênero gramatical do suposto antecedente. Nesses experimentos, o processamento de pronomes cujo antecedente é comum de dois gêneros mas não há informação quanto ao gênero do mesmo pelo artigo, como em l'amante, foi tomado como linha de base, tendo em vista que, nesse caso, o gênero do pronome determina o gênero do antecedente. $\mathrm{O}$ tempo de leitura obtido nessa condição foi semelhante ao tempo de leitura obtido na condição em que o gênero do pronome não corresponde ao gênero do antecedente.

Os resultados de Cacciari, Carreiras e Cionini (1997), quando interpretados à luz do tipo de informação que a língua disponibiliza na interface, podem sugerir que informação léxico-sintática relativa ao gênero gramatical do antecedente foi levada em conta no processamento da forma pronominal, independentemente de o antecedente encontrar-se ou não na sentença que a contém, dado que menor tempo de leitura foi obtido no processamento de formas pronominais nominativas e clíticos cujo gênero concorda como o gênero do antecedente [+ animado].

Por outro lado, o fato de o tempo de leitura na condição em que o gênero do pronome não coincide com o do antecedente ter sido semelhante ao obtido na condição em que o gênero do pronome determina o gênero do antecedente pode ser indicativo de que a presença de antecedente [+ animado] viabiliza a interpretação semântica do gênero do pronome. $\mathrm{O}$ gênero do pronome, semanticamente interpretado, permitiria a atribuição do referente do antecedente lingüístico a uma classe natural de gênero.

Os resultados relativos a nomes próprios, por sua vez, podem ser indicativos de ausência de pareamento de traços entre pronome e antecedente. Diferentemente do pronome em (1), o nome próprio em (2) não é uma expressão anafórica. Pelo contrário, este tende a introduzir novo referente, a reintroduzir referente já mencionado quando a expressão que o introduziu já não se encontra acessível ao falante/ouvinte, ou simplesmente serve para demarcar progressão discursiva (Ariel 1994; Corrêa 1993). Logo, o nome próprio não promoveria reativação de possíveis antecedentes com base na qual seria feito um pareamento de traços léxico-sintáticos. Como explicar, então, o efeito de concordância semelhante ao encontrado com formas pronominais? Nesse caso, tomar um elemento [+ animado] que compartilha o gênero gramatical do nome próprio como co-referente deste último seria uma estratégia para a integração semântica das proposições apresentadas. Se o mesmo ocorrer com formas pronominais, ou seja, se o gênero do pronome semanticamente interpretado for atribuído ao referente do antecedente lingüístico [+ animado] que com este compartilha o traço formal de gênero, tem-se a atribuição do sexo feminino ao referente de la vittima.

(1) La vittima dell'incidente stradale sbatté violentamente la testa contra il finestrino. Lei/Lui, perció, parse molto sangue e svenne. 
(2) La vittima dell'incidente stradale sbatté violentamente la testa contra il finestrino. Maria/Mario, perció, parse molto sangue e svenne.

Em suma, os resultados de Cacciari, Carreiras e Cionini (1997) não deixam claro se o a interpretação do gênero do pronome interfere no processamento do que se apresenta como concordância pronominal.

\section{EXPERIMENTOS}

Experimento 1:

Este experimento visa a verificar se o traço de gênero do pronome é semanticamente interpretado e de que modo essa interpretação afetaria o processamento da referência pronominal dependente de informação de natureza léxico-sintática relativa a gênero.

Congruência entre o gênero gramatical do pronome e o gênero natural do referente do antecedente lingüistico é tomada como variável independente. Assume-se que o antecedente (DP) em posição inicial na sentença, com traço semântico [+animado/+ humano] é o mais acessível para a resolução da referência pronominal (Ariel 1990; Corrêa 1993; 2000). No caso de perda de informação lexico-sintática, a representação conceptual correspondente ao elemento [+animado] ou ao referente do sujeito/tópico da primeira sentença do par seria, conseqüentemente, a mais acessível.

Três condições experimentais são criadas: Congruente, Incongruente e Neutra. Em todas as condições, a concordância entre o gênero gramatical do pronome e o do antecedente mais acessível é mantida. Na condição Congruente, o gênero gramatical do pronome semanticamente interpretado seria compatível com o gênero natural atribuído (por inferência) ao referente do antecedente mais acessível, como em (3). Na condição Incongruente, o gênero gramatical do pronome semanticamente interpretado seria incompatível com o gênero natural atribuído (por inferência) ao referente do antecedente mais acessível, como em (4). Na condição Neutra, não há informação adicional que permita atribuir o referente do antecedente mais acessível a uma categoria natural de gênero, como em (5).

Essa condição foi incluída como linha de base para o processamento da referência pronominal baseado exclusivamente em informação léxico-sintática relativa ao gênero do antecedente mais acessível.

(3) A testemunha de meia-calça preta/ narrou a briga violenta/ na sala do comissário/ por meia hora./ Ela T1/ falava T2/ com nervosismo.T3/ Uma mulher falava nervosamente/.

(4) A testemunha de barba preta/ narrou a briga violenta /na sala do comissário /por meia horal. Ela T1/falava T2/ com nervosismoT3./ Um homem falava nervosamente. 
(5) A testemunha de roupas claras/ narrou a briga violenta/ na sala do comissário/ por meia hora./ Ela T1/falava T2/ com nervosismo./ A testemunha falava nervosamente

A tarefa utilizada é a de leitura auto-monitorada seguida de julgamento do valor verdade da proposição final. $\mathrm{O}$ julgamento serve apenas para garantir a compreensão do texto lido. Os pares de sentença foram semelhantes aos utilizados em Cacciari, Carreiras e Cionini (1997), exceto pela informação adicional trazida por um adjunto. Ainda diferentemente de Cacciari, Carreiras e Cionini (1997), neste experimento, o pronome sempre concorda com o gênero gramatical do antecedente mais acessível ${ }^{3}$.

As barras em (3-5) delimitam os segmentos apresentados na tela de um computador para leitura. Cada segmento permanece na tela apenas durante o tempo em que é lido. A introdução de novo segmento causa o apagamento do anterior. Os pontos T1, T2 e T3 indicam os momentos em que o tempo de leitura é mensurado.

Parte-se da hipótese de que a interpretação semântica do gênero do pronome afeta o processamento da referência pronominal. Dois tipos de previsão podem ser feitos em função da disponibilidade de informação léxico-sintática relativa ao gênero do antecedente na memória de trabalho:

(a) a informação relativa ao gênero gramatical do antecedente não se mantém disponível na memória de trabalho e a referência pronominal é feita diretamente à representação conceptual do referente do antecedente lingüístico.

- Prevê-se menor tempo de leitura para a condição Congruente em relação à condição Incongruente, dada a impossibilidade de a referência pronominal se estabelecer com a representação conceptual de um indivíduo do sexo masculino;.

- Prevê-se tempo de leitura igual ou maior na condição Neutra em relação à Incongruente dada a ausência de informação semântica e léxico-sintática relativa a gênero na memória de trabalho - tem-se a representação de um indivíduo, de sexo indeterminado, com determinada propriedade, como ser vítima, testemunha, etc., na memória de trabalho.

Tem-se assim: Congruente $<$ Neutra $\leq$ Incongruente.

(b) a informação relativa ao gênero gramatical do antecedente se mantém disponível na memória de trabalho:

- Prevê-se menor tempo de leitura para a condição Congruente em relação à Neutra, dado que, na primeira, a atribuição da interpretação do gênero do pronome ao referente do antecedente lingüístico seria corroborada pela informação adicional relativa ao sexo do referente - indivíduo do sexo feminino/masculino, enquanto que na segunda, a atribuição da interpretação do gênero do pronome ao referente

\footnotetext{
${ }^{3}$ Um terceiro experimento da série varia o gênero da forma pronominal
} 
teria de ser avaliada em função do gênero do antecedente, o qual é semanticamente indeterminado.

- Prevê-se menor tempo de leitura na condição Incongruente do que na condição Neutra, uma vez que a interpretação do gênero do pronome seria imediatamente descartada diante de informação adicional relativa ao sexo do referente.

- Quanto à diferença entre Congruente e Incongruente, considera-se que a primeira acarretaria maior custo de processamento dada a integração de diferentes fontes de informação - informação de natureza léxico-sintática relativa a gênero, informação de natureza semântica relativa ao gênero do pronome e informação de natureza conceptual relativa ao sexo do referente do antecedente lingüístico. $\mathrm{Na}$ segunda, não haveria integração de informação, uma vez descartada a interpretação semântica do pronome.

Tem-se assim: Incongruente < Congruente < Neutra.

Quanto ao uso de informação conceptual no processamento da referência pronominal, modelos que incorporam componentes modulares fariam prever um efeito de informação de natureza conceptual em T3. Modelos de natureza incremental fariam prever esse efeito a partir de $\mathrm{T} 1$.

Sujeitos:

Os sujeitos foram 22 estudantes de graduação e de pós-graduação da PUC-Rio (10 do sexo masculino), na faixa etária de 18 a 42 anos, que participaram como voluntários.

Material:

O material lingüístico constou de: (i) Dois conjuntos de seis pares de sentenças por condição, sendo metade com pronome e antecedente de gênero masculino e metade com pronome e antecedente de gênero feminino. Em todas os pares, o antecedente mais acessível (em posição inicial na sentença), com traço [+ animado] e/ou [+ humano], é invariável quanto ao gênero. Cada conjunto contém três tipos de pares de sentenças que são idênticos entre condições, exceto pela informação apresentada pelo adjunto. Para cada sujeito experimental, há um total de seis diferentes pares de sentenças-teste, com dois pares por condição; (ii) Um conjunto de dezoito distratores, contendo pares de sentenças com oração relativa encaixada no sujeito.

Como equipamento, tem-se: um monitor Samsung 15" e uma caixa de respostas com três botões ( $\mathrm{C}$, para continuar, $\mathrm{V}$ para verdadeiro e $\mathrm{F}$ para falso), ambos conectados a um computador Pentium 166 PC.

Procedimento:

Os sujeitos foram convidados a participar de um estudo sobre compreensão na leitura. O procedimento de testagem foi inicialmente explicado oralmente pelo experimentador. Os sujeitos foram testados individualmente numa cabine com atenuação acústica do Laboratório de Psicolinguística da PUC-Rio, na qual se encontram o monitor e a caixa de respostas. A sessão experimental consistiu de 3 fases: 
leitura de instruções, treinamento e teste. Os sujeitos foram instruídos a lerem de forma natural os fragmentos de sentença apresentados e a pressionarem o botão C tão $\operatorname{logo}$ terminassem a leitura do mesmo. Na fase de treinamento, três pares de sentenças, semelhantes aos pares de teste e aos distratores foram apresentados, seguidos de uma sentença declarativa completa para julgamento do valor verdade, mediante pressão no botão $\mathrm{V}$ ou no $\mathrm{F}$. $\mathrm{O}$ treinamento pôde ser repetido conforme a vontade do sujeito. Iniciada a fase de teste, os pares de sentenças testes e distratores foram apresentados na tela do monitor em ordem aleatória por sujeito, sendo que a apresentação consecutiva de pares da mesma condição experimental foi evitada. Os pares de sentenças foram apresentados em segmentos correspondentes a sintagmas seguidos da proposição para julgamento, apresentada por inteiro. A apresentação dos segmentos de texto na tela do monitor foi feita mediante pressão no botão $C$ pelo sujeito experimental. Uma vez acionado o botão, o segmento recém-lido era apagado e substituído pelo subseqüente. A pressão no botão $\mathrm{C}$ nos pontos críticos (T1, T2 e T3) permite que o tempo de leitura seja cronometrado. A apresentação dos estímulos no monitor, a mensuração do tempo de leitura e seu registro foram feitos por meio de programa elaborado em linguagem EXPE6 (Pallier, Dupoux \& Jeannin 1999).

Resultados:

Uma análise da variância (3-way) com congruência como medida repetida foi realizada para cada variável dependente, a saber, os tempos de leitura nos pontos $\mathrm{T} 1$, T2 e T3 . O valor limite (cut-off) para os tempos de leitura em T1 e T2 foi fixado em $1000 \mathrm{mseg}$. A tabela 1 apresenta as médias obtidas.

\begin{tabular}{llll}
\hline & & Congruência & \\
Ponto & Neutra & Congruente & Incongruente \\
T1 & 506.25 & 505.91 & 481.90 \\
T2 & 571.79 & 505.97 & 501.72 \\
T3 & 958.61 & 874.50 & 805.59 \\
\hline
\end{tabular}

Tabela 1: Média dos tempos de leitura (mseg) em função de congruência

Os resultado obtidos em T1 não revelam efeito significativo de congruência $(\mathrm{p}=.7)$. Em T2 e T3 há um efeito marginalmente significativo: $\mathrm{F}(2,18)=3,39$ $\mathrm{p}=.05$ em T2 e $\mathrm{F}(2,21)=3,16 \mathrm{p}=.05$ em T3.

A direção das médias em T2 aponta para maior dificuldade no processamento na condição neutra em relação às demais, que não diferem entre si. Em T3, o maior tempo de resposta continua ocorrendo na condição Neutra. Nesse ponto, contudo, menor tempo é obtido da condição Incongruente.

Discussão:

Os resultados obtidos são compatíveis com a hipótese de que a interpretação semântica do gênero do pronome afeta o processamento da referência pronominal e estão de acordo com as previsões em (b), que têm como pressuposto a disponibilidade 
de informação léxico-sintática relativa ao gênero do antecedente lingüístico na memória de trabalho. O efeito obtido em T2 sugere que informação semântica adicional foi buscada após o verbo embora a compatibilização desta com o gênero do pronome só tenha sido efetuada em T3.

Experimento 2:

O presente experimento visa a dificultar a recuperação de informação léxicosintática relativa ao gênero do antecedente pretendido quando do processamento da forma pronominal de modo a verificar se, na ausência de informação léxico-sintática, a referência pronominal seria resolvida a partir da interpretação semântica do traço de gênero do pronome. Para isso, uma sentença com duas orações coordenadas é introduzida entre as sentenças dos pares utilizados no Experimento 1 (cf. (6-8)). Se, na ausência de informação léxico-sintática, a referência pronominal se resolver a partir de uma correspondência entre a interpretação semântica do traço de gênero do pronome e a representação conceptual do referente do DP-sujeito da primeira oração, então as previsões expressas em (a) no experimento 1 deverão ser verificadas.

Tal como no Experimento 1, três medidas de tempo de leitura são tomadas — T1, após o pronome; T2, após o verbo e T3, ao fim da sentença. Busca-se verificar com essas medidas se, diante da ausência de informação léxico-sintática relativa ao gênero do antecedente lingüístico, informação semântica seria levada em conta em momentos iniciais do processamento da sentença.

Condição congruente:

(6) A testemunha de meia-calça preta/narrou a briga violenta/ na sala do comissário/ por meia hora./O aposento/ era abafado/ e papéis/se amontoavam./ Ela T1/falava $\mathrm{T} 2 /$ com nervosismo. RT3/ Uma mulher falava nervosamente./

Condição não congruente:

(7) A testemunha de barba preta/ narrou a briga violenta /na sala do comissário /por meia hora/. O aposento/ era abafado/ e papéis/ se amontoavam./ Ela T1/falava T2/ com nervosismo./T3. Um homem falava nervosamente./

Condição neutra:

(8) A testemunha de roupas claras / narrou a briga violenta/ na sala do comissário/ por meia hora./O aposento/ era abafado/ e papéis/se amontoavam./ Ela T1/falava $\mathrm{T} 2 /$ com nervosismo./T3 A testemunha falava nervosamente./ 
Método:

Sujeitos:

Os sujeitos foram 26 alunos de graduação e de pós-graduação da PUC-Rio (11 do sexo masculino), de faixa etária semelhante à do Experimento 1, que não haviam participado daquele.

Material e procedimento: Idênticos ao do experimento 1, exceto pela inclusão da sentença coordenada entre as sentenças do par crítico do Experimento 1 e por alterações nos distratores de modo a controlar o tamanho dos textos apresentados. $\mathrm{O}$ gênero dos nomes das orações coordenadas foi controlado de modo a não coincidir com o gênero da forma pronominal e nomes com traço [+ animado] não foram utilizados nessas orações.

Resultados:

Assim como no Experimento 1, uma análise da variância (3-way) com congruência como medida repetida foi realizada para cada variável dependente, a saber, os tempos de leitura nos pontos T1, T2 e T3. O valor limite para os tempos de leitura em T1 e T2 foi fixado em 1000 mseg. A tabela 2 apresenta as médias obtidas.

\begin{tabular}{llll}
\hline & & $\begin{array}{l}\text { Congruência } \\
\text { Congruente }\end{array}$ & Incongruente \\
Ponto & Neutra & 498,8 & 483,34 \\
T2 & 551,14 & 455,83 & 503,35 \\
T3 & 521,27 & 688,71 & 816,52 \\
\hline
\end{tabular}

Tabela 2: Média dos tempos de leitura (mseg) em função de congruência

Obteve-se um efeito significativo de Congruência nos três pontos considerados: T1, imediatamente após o pronome $\mathrm{F}(2,24)=4,87 \mathrm{p}=.01$; T2, após o verbo $\mathrm{F}(2,23)=3,99 \mathrm{p}<.03$ e T3, ao fim da sentença, $\mathrm{F}(2,26)=3,42 \mathrm{p}<.05$. Um teste post hoc foi conduzido entre as condições congruente e incongruente no ponto 1 e no ponto 2 e entre as condições neutra e incongruente no ponto 3 e não foram obtidos efeitos significativos. Assim sendo, o efeito de Congruência obtido em T1 e em T2 deve-se à diferença entre a condição Neutra e as demais. Em T3, o efeito deve-se à vantagem da condição Congruente sobre as Condições Neutra e Incongruente.

Discussão:

Esses resultados são compatíveis com as previsões para o efeito da interpretação do gênero do pronome no processamento da referência pronominal quando da ausência de informação léxico-sintática relativa ao gênero do antecedente lingüístico. $O$ efeito de congruência em T1 e em T2 sugere que, na ausência de informação de natureza léxico- 
sintática, informação semântica é buscada imediatamente após o pronome. O efeito em T3 sugere, não obstante, que essa informação conceptual só é levada em conta no processamento da referência pronominal ao fim da sentença.

\section{Comparação dos resultados}

Os resultados dos dois experimentos foram comparados de modo a verificar-se o efeito da distância entre antecedente e pronome sobre os resultados. Uma análise da variância foi conduzida para cada variável dependente - T1, T2 e T3. A distância entre antecedente e pronome foi tomada como fator grupal no design 2 (distância) X 3 (congruência).

Os resultados apresentam um efeito significativo da interação entre distância e congruência em T3: $\mathrm{F}(2,92)=4,34 \mathrm{p}<.05$. A tabela 3 apresenta as médias.

\begin{tabular}{llll}
\hline & & Congruência & \\
& Neutra & Congruente & Incongruente \\
Distância 1 & 958,61 & 874,50 & 805,59 \\
Distância 2 & 769,90 & 688,71 & 816,51 \\
\cline { 2 - 4 } Média Total & 879,57 & 758,37 & 813,92 \\
\hline
\end{tabular}

Tabela 3: Média dos tempos de leitura (mseg) em função de congruência e distância em T3

Discussão:

O efeito da interação entre distância e congruência deve-se ao fato de o tempo de leitura na distância 2 ter sido menor do que na distância 1 nas condições Neutra e Congruente, enquanto que não há diferença em função da distância na condição Incongruente. Assumindo-se que a maior distância dificulta ou impede o acesso à informação de natureza léxico-sintática relativa a gênero, os maiores tempos de leitura nas condições Neutra e Congruente na distância 1 podem ser atribuídos à sobrecarga na memória decorrente da manutenção dessa informação ao longo do processamento da sentença que contém a forma pronominal. O tempo obtido nas duas distâncias na condição Incongruente parece indicar que o custo do processamento é semelhante quando da ausência de informação semântica compatível com a interpretação semântica do gênero do pronome, independentemente da disponibilidade de informação léxicosintática. Parece, pois, que a interpretação semântica do gênero do pronome é parte do processamento do que se apresenta como concordância pronominal.

Discussão final e conclusão

Os resultados obtidos sugerem que o processamento da concordância de gênero em formas pronominais faz uso de informação de natureza léxico-sintática relativa ao gênero gramatical do antecedente mais acessível e que a resolução da referência se dá ao fim da sentença, quando diferentes fontes de informação podem ser compatibilizadas. Os resultados são, portanto, compatíveis com a proposta de modelos 
modulares de processamento que prevêem um pareamento automático de traços léxicosintáticos, anterior à recuperação de informação semântica e processos integrativos tardios (Friederici 1995). Informação léxico-sintática relativa ao gênero gramatical no processamento de formas pronominais no discurso viria garantir a coesão entre sentenças independentes. De qualquer forma, a possibilidade de o gênero do pronome ser semanticamente interpretado contribui para a resolução da referência, tornando o processamento menos custoso quando há informação adicional compatível com essa interpretação. Os resultados sugerem ainda que é possível que a referência pronominal venha a ser resolvida em bases semânticas, a partir do gênero do pronome semanticamente interpretado, quando da não disponibilidade de informação léxicosintática relativa ao antecedente lingüístico. Em suma, a concordância pronominal parece ser dependente de condições de processamento específicas que garantam acesso a informação léxico-sintática relativa ao gênero do antecedente, ainda que a interpretação semântica do gênero do pronome possa viabilizar a referência pronominal na ausência daquela.

Esse estudo buscou uma aproximação entre a investigação psicolingüística sobre processamento de relações de concordância em sentido amplo e uma teoria de língua, considerando a noção de interpretabilidade de traços formais na interface como a possibilidade de leitura e interpretação semântica de informação lingüística por sistemas de desempenho. Ainda que este conceito não esteja suficientemente explicitado no âmbito da teoria lingüística, a aproximação entre modelos de língua e de processamento em função do mesmo parece ser promissora.

\section{REFERÊNCIAS}

ARIEL, M. (1994). Interpreting anaphoric expressions: A cognitive vs a pragmatic approach. Linguistics, 30: $3-42$.

ARNOLD, J. E., EISENBAND, J. G., BROWN-SCHMIDT, S. \& TRUESWELL, J. C. (2000). The rapid use of gender information: evidence of the time course of pronoun resolution from eyetracking. Cognition, 00, 1-14.

CACCIARI, C., CARREIRAS, M. \& CIONINI, C.B. (1997). When words have two genders: Anaphor resolution for Italian functionally ambiguous words. Journal of Memory and Language, 37, 517-532.

CARREIRAS, M., GARNHAM, A. \& OAKHILL, J. (1993). The use of superficial and meaning-based representations in interpreting pronouns. Evidence from Spanish. European Journal of Cognitive Psychology, 5, 1, $93-116$.

CHOMSKY, N. (1995). The Minimalist Program. Cambridge, Mass: MIT Press.

COMRIE, B. (1999). Grammatical gender systems: A linguist's assessment. Journal of Psycholinguistic Research, vol.28, $\mathrm{n}^{\circ}$ 5, 457-466.

CORBETT, G. (1991). Gender. Cambridge. CUP.

CORRÊA, L.M.S. (1993). Restrições ao pronome livre na linearização do discurso. Revista PaLavra, no 1 , 75-95. 
. (2000). Acessibilidade diferenciada e fatores estruturais na produção e na compreensão de formas pronominais. Revista Palavra, nº 6, 134-153.

CRAWLEY, R. A., STEVENSON, R. J. \& KLEIMAN, D. (1990). The use of heuristic strategies in the interpretation of pronouns. Journal of Psycholinguistic Research, 19, 245-264.

DEUTSCH, A. \& BENTIN, S. (2001). Syntactic and semantic factors in processing gender agreement in Hebrew: evidence from ERPs and eye movements. Journal of Memory and Language, 45, 200-224.

FRIEDERICI, (1995). The time course of syntactic activation during language processing: A model based on neuropsychological and neurophysiological data. Brain and Language, 50, 259-281.

FRIEDERICI, A. \& JACOBSEN, T. (1999). Processing grammatical gender during language comprehension. Journal of Psycholinguistic Research, vol.28, no.5, 467-486.

FRIEDERICI, A., MECKLINGER, A., SPENCER, K. M., STEINHAUER, K. \& DONCHIN, E. (2001). Syntactic parsing preferences and their on-line revisions: a spatio-temporal analysis of event-related brain potentials. Cognitive Brain Research,11, 2, 305-323.

GARNHAM, A., OAKHILL, J., EHRLICH, M.F. \& CARREIRAS, M. (1995). Representation and Processes in the Interpretation of Pronouns: New Evidence from Spanish and French. Journal of Memory and Language, 34, 41-62.

GERNSBACHER, M. A. (1991). Comprehending conceptual anaphors. Language and Cognitive Processes, 6, 81- 105 .

GORDON, P. C. \& CHAN, D. (1995). Pronouns, passives and discourse coherence. Journal of Memory and Language, 34, 216-231.

GROSZ, B. J. \& SIDNER, C. L. (1986). Attention, intentions, and the structure of discourse. Computational Linguistics, 12, 175-204.

GUNTER, T.C., FRIEDERICI, A.D. \& SCHRIEFERS, H. (2000). Syntactic gender and semantic expectancy: ERPs reveal early autonomy and late interaction. Journal of Cognitive Neuroscience, 12, $556-568$.

HAHNE, A. \& FRIEDERICI, A. (2000). Differential task effects on semantic and syntactic processes as revealed by ERPs. Cognitive Brain Research ,13, 3, 339-356.

JARVELLA, R. J. \& HERAMN, S. J. (1972). Clause structure of sentences and speech processing. Perception and Psychophysics, 11, 381-384.

LIU, S. R. A., CHIARELLO, C. \& QUAN, N. (1999). Hemispheric sensitivity to grammatical cues: evidence for bilateral processing of number agreement in noun phrases. Brain and Language, 70, 483-503.

MCKOON, G., GREENE, S. B. \& RATCLIFF, R. (1993). Discourse models, pronoun resolution, and the implicit causality of verbs. Journal of Experimental Psychology: Learning, Memory and Cognition, $19,1040-1052$.

PALLIER, C., DUPOUX, E. \& JEANNIN, X. (1999). EXPE6 - Reference Manual. Laboratoire de Sciences Cognitives et Psycholinguistique, EHHSS, CNRS.

PEARLMUTTER, N. J., GARNSEY, S. M. \& BOCK, K. (1999). Agreement processes in sentence comprehension. Journal of Memory and Language, 41, 427-456. 
SCHRIEFERS, H. \& JESCHENIAK, J.D. (1999). Representation and processing of grammatical gender in language production: A review. Journal of Psycholinguistic Research, vol.28, no. 6, 575-600.

TOWNSEND, D. J. \& BEVER, T.G. (1978). Interclause Relations and Clausal Processing. Journal of Verbal Learning and Verbal Behavior, 17, 509-521.

VAN BERKUN, J.J.A., BROWN, C. \& HAGOORT, P. (1999). When does gender constrain parsing? Evidence from ERP. Journal of Psycholinguistic Research, vol.28, no.5, 555-571. 